\title{
Neurovegetative symptom subtypes in young people with major depressive disorder and their structural brain correlates
}

\author{
Yara J. Toenders $\mathbb{1}^{1,2}$, Lianne Schmaal ${ }^{1,2}$, Ben J. Harrison ${ }^{3}$, Richard Dinga ${ }^{4,5}$, Michael Berk ${ }^{1,2,6,7,8}$ and \\ Christopher G. Davey ${ }^{1,2}$
}

\begin{abstract}
Depression is a leading cause of burden of disease among young people. Current treatments are not uniformly effective, in part due to the heterogeneous nature of major depressive disorder (MDD). Refining MDD into more homogeneous subtypes is an important step towards identifying underlying pathophysiological mechanisms and improving treatment of young people. In adults, symptom-based subtypes of depression identified using data-driven methods mainly differed in patterns of neurovegetative symptoms (sleep and appetite/weight). These subtypes have been associated with differential biological mechanisms, including immuno-metabolic markers, genetics and brain alterations (mainly in the ventral striatum, medial orbitofrontal cortex, insular cortex, anterior cingulate cortex amygdala and hippocampus). K-means clustering was applied to individual depressive symptoms from the Quick Inventory of Depressive Symptoms (QIDS) in 275 young people (15-25 years old) with MDD to identify symptombased subtypes, and in 244 young people from an independent dataset (a subsample of the STAR*D dataset). Cortical surface area and thickness and subcortical volume were compared between the subtypes and 100 healthy controls using structural MRI. Three subtypes were identified in the discovery dataset and replicated in the independent dataset; severe depression with increased appetite, severe depression with decreased appetite and severe insomnia, and moderate depression. The severe increased appetite subtype showed lower surface area in the anterior insula compared to both healthy controls. Our findings in young people replicate the previously identified symptom-based depression subtypes in adults. The structural alterations of the anterior insular cortex add to the existing evidence of different pathophysiological mechanisms involved in this subtype.
\end{abstract}

\section{Introduction}

Approximately 322 million people worldwide (5\% of the world's population) suffer from Major Depressive Disorder (MDD); a disease characterized by a depressed mood and associated symptoms ${ }^{1}$. In young people, depressive disorders are the main cause of global burden of disease ${ }^{2}$. The onset of MDD peaks during adolescence

\footnotetext{
Correspondence: Lianne Schmaal (lianne.schmaal@unimelb.edu.au)

'Orygen, The National Centre of Excellence in Youth Mental Health, Parkville,

VIC, Australia

${ }^{2}$ Centre for Youth Mental Health, The University of Melbourne, Parkville, VIC, Australia

Full list of author information is available at the end of the article

These authors contributed equally: Yara J. Toenders, Lianne Schmaal
}

and young adulthood, and earlier onset of MDD is associated with decreased quality of life and increased impairment in social and occupational functioning later in life $^{3,4}$. Currently available treatments are not uniformly effective for adolescent depression, with response rates around $61 \%$ for antidepressants and 55\% for psychother$\mathrm{apy}^{5,6}$. The unpredictable nature of treatment response might be explained, at least in part, by the heterogeneity of MDD.

The most commonly used systems for classifying mental disorders, the ICD 10 (International Classification of Diseases 10th revision) and Diagnostic and Statistical Manual of Mental Disorders (DSM-5), categorize a broad

\section{(c) The Author(s) 2020}

(c) (i) Open Access This article is licensed under a Creative Commons Attribution 4.0 International License, which permits use, sharing, adaptation, distribution and reproduction in any medium or format, as long as you give appropriate credit to the original author(s) and the source, provide a link to the Creative Commons license, and indicate if changes were made. The images or other third party material in this article are included in the article's Creative Commons license, unless indicated otherwise in a credit line to the material. If material is not included in the article's Creative Commons license and your intended use is not permitted by statutory regulation or exceeds the permitted use, you will need to obtain permission directly from the copyright holder. To view a copy of this license, visit http://creativecommons.org/licenses/by/4.0/. 
spectrum of depressive symptom patterns within a single MDD diagnosis. To receive an MDD diagnosis, a minimum of 5 of the 9 DSM criteria for MDD have to be met. Considering that some of the criteria include symptoms of opposite polarity (e.g. increased versus decreased appetite, weight gain versus loss, insomnia versus hypersomnia, and psychomotor agitation versus retardation), almost 1500 different combinations of MDD symptoms lead to the same DSM diagnosis of $\mathrm{MDD}^{7}$. Thus, patients with the same diagnosis show heterogeneous depressive symptom profiles, which may reflect different underlying neurobiological mechanisms that could require different treatments. Identifying more homogeneous subtypes of depression could inform clinical judgement and guide treatment selection.

Several attempts to identify subtypes of depression have been made to overcome these issues associated with the traditional diagnostic classification and the heterogeneity of MDD. Traditionally, subtypes of depression have been defined based on subjective expert consensus. An example of describing different subtypes are the DSM atypical and melancholic depression specifiers ${ }^{1}$. The atypical specifier is characterized by mood reactivity in combination with increases in weight or appetite, hypersomnia and/or leaden paralysis. The melancholic specifier is distinguished by opposite neurovegetative symptoms: decreases in weight or appetite and early morning awakening, in addition to psychomotor agitation or retardation, worse mood in the morning and excessive feelings of guilt. Existence of the melancholic specifier has been confirmed by prior research; however, the atypical specifier has been questioned. For example, mood reactivity, the only obligatory atypical symptom in the DSM, does not show associations with the other atypical features ${ }^{8,9}$. More recently, data-driven approaches have been employed to identify symptom-based depression subtypes. Replicated across a number of studies in adults, latent class analysis has derived data-driven typical versus atypical neurovegetative symptom subtypes ${ }^{10-17}$. The typical and atypical subtypes are usually characterized as having similar affective and cognitive symptoms, differing only on sleep and appetite profiles. Of note, the atypical neurovegetative symptom subtype differs from the atypical specifier in the DSM as it mainly shows only reversed neurovegetative symptoms (i.e., increased appetite and weight, and in some studies also hypersomnia). However, not much is known about whether similar subtypes exist in young people with depression, although one study suggests they are similar to adults ${ }^{18}$.

Importantly, there are biological differences between the previously found data-driven neurovegetative symptom subtypes in adults. Higher levels of leptin, inflammatory markers (C-reactive protein (CRP), interleukin-6 (IL-6), interleukin 1 receptor antagonist (IL-1RA) and tumor necrosis factor- $\alpha$ (TNF- $\alpha)$ ), insulin and higher BMI are associated with the atypical or increased appetite subtype ${ }^{15,19-21}$. Conversely, higher cortisol and ghrelin levels are associated with the more typical subtype characterized by decreased appetite ${ }^{19,21-23}$. In addition, genetic studies have shown that the atypical subtype with increased appetite is associated with a higher polygenic risk score for BMI, leptin and CRP, whereas the typical subtype showed a stronger association with polygenic risk scores for psychiatric disorders such as schizophrenia ${ }^{24,25}$. Moreover, brain activation responses to pictures of food have been shown to differentiate depressed patients selected on having either increased or decreased appetite ${ }^{21,26}$. Higher cortisol levels in the MDD group with decreased appetite were negatively correlated with ventral striatal activity during a food task; while the MDD group with increased appetite showed a positive correlation between insulin resistance and posterior and dorsal mid-insula cortex activity $^{21}$. This increased appetite group showed higher anterior insula cortex activity in response to these appetitive food pictures ${ }^{26}$. In addition, high levels of the inflammatory marker CRP were associated with higher coupling between anterior insula and orbitofrontal cortex (OFC) and ratings of food pleasantness in the MDD group with increased appetite ${ }^{27}$. Other brain regions implicated in neurovegetative appetitive symptoms are part of the salience network, such as the rostral anterior cingulate cortex (ACC) and amygdala ${ }^{28-30}$. In addition, the hippocampus is thought to be affected by appetitive hormones and thereby have a role in appetite inhibition ${ }^{31}$.

There is now consistent evidence in adults for different biological correlates across neurovegetative symptom subtypes, however, it remains unknown whether similar subtypes exist in young people and if they are characterized by similar biological mechanisms. The current study aims to replicate the data-driven symptom profiles based on neurovegetative symptoms, previously identified in adults, in young people with MDD. Furthermore, we included an additional independent sample as a replication cohort. In addition, the study aims to examine structural brain alterations associated with the identified subtypes. We hypothesize that similar subtypes exist in young people, mainly distinguished by opposite neurovegetative symptoms. We also hypothesize that structural alterations in subregions of the insula, ventral striatum, medial OFC, ACC, amygdala and hippocampus may differentiate between the symptom subtypes, since these regions have been implicated in subtype differences in previous adult studies ${ }^{21,26}$ or more generally associated with neurovegetative appetite symptoms (representing the most discriminating symptoms between the previously identified data-driven subtypes). 


\section{Methods and materials Participants Discovery sample}

Participants were recruited from youth mental health centers in Australia as part of the YoDA-A and YoDA-C (Youth Depression Alleviation) studies ${ }^{32,33}$. In total, 275 young people with MDD and 100 age and sex-matched healthy controls $(\mathrm{HC})$ were included. All participants were aged between 15 and 25 years old and the participants in the MDD group were diagnosed with a primary diagnosis of MDD. For the MDD participants, a score of 20 or higher on the Montgomery-Åsberg Depression Rating Scale (MADRS), indicating at least moderate severity of symptoms, was required. The $\mathrm{HC}$ participants were recruited through advertisements and did not have a present or past diagnosis of MDD or anxiety disorders. Participants were excluded if they suffered from an acute medical disorder, had experienced any psychotic episodes, or were diagnosed with bipolar disorder. Further, pregnancy, breastfeeding and any contraindications to MRI were exclusion criteria. The participants gave written informed consent and the Melbourne Health Human Research Ethics Committee approved the study protocol.

\section{Replication sample}

The data for the independent replication sample came from the STAR*D study, a large multicenter study examining antidepressant effectiveness ${ }^{34}$. To match the YoDA sample, the sample was restricted to young people between 18 and 25 years old with an MDD diagnosis, resulting in a sample size of 244 . We used the baseline data for participants in the STAR*D study. All participants scored 14 or higher on the Hamilton Depression Rating Scale (HDRS) ${ }^{35}$, indicating moderate to severe depression. Exclusion criteria were a primary diagnosis of schizophrenia, bipolar disorder, anorexia nervosa, bulimia or obsessive-compulsive disorder. In addition, the participants were free of antidepressants when they entered the study. The STAR"D sample is comparable to the YoDA sample in age, gender and depression severity. In both studies, only participants with moderate to severe depression based on either the Montgomery-Åsberg Depression Rating Scale or the Hamilton Depression Rating Scale were included. The total QIDS score (at study inclusion) was highly similar between both samples across the subtypes (Tables 1,2). The participants in STAR*D were not using antidepressants at time of inclusion and similarly only a small proportion (7\%) of the YoDA participants were using antidepressants at time of inclusion.

\section{Procedure \\ Discovery sample}

The YoDA participants were screened using the Structured Clinical Interview for the DSM-IV (SCID) during the baseline assessment ${ }^{34}$. In addition, depressive symptoms were measured using the MADRS as well as the Quick Inventory of Depressive Symptomatology Self Report (QIDS-SR $)^{36,37}$. Further, participants completed the Generalized Anxiety Disorder 7 questionnaire (GAD-7), Social and Occupational Functioning Assessment Scale (SOFAS), Alcohol Use Disorder Identification Test $(\text { AUDIT) })^{38-40}$ and other questionnaires not germane to this study. Within 2 weeks of the baseline assessment, and prior to commencing the study treatments, a subset of the participants who chose to participate in the nonobligatory MRI assessment and were not excluded based on MRI eligibility criteria (137 MDD patients and 100 healthy controls) underwent a structural MRI scan.

\section{Replication sample}

Baseline scores of the QIDS of STAR*D participants were included in this study ${ }^{36}$. The Psychiatric Diagnostic Screening Questionnaire (PDSQ) was used as a diagnostic screening tool in the STAR*D study ${ }^{41}$. Quality of life was assessed with the Quality of Life Enjoyment and Satisfaction Questionnaire (Q-LES-Q) and impaired functioning using the Work and Social Adjustment Scale $(\text { WSAS })^{42,43}$. No MRI data were available in this sample.

\section{MRI data}

\section{Discovery sample}

The T1 weighted scan lasted $\sim 4 \mathrm{~min}$ and was performed on a $3 \mathrm{~T}$ General Electric Signa Excite at Sunshine Hospital (Western Health, Melbourne). An 8-channel phasedarray head coil was used (repetition time (TR): $7900 \mathrm{~ms}$, echo time (TE): $3000 \mathrm{~ms}$, TI: 400, thickness (no gap): 1 $\mathrm{mm}$, flip angle: $13^{\circ}$, field of view: $25.6 \mathrm{~cm}$, pixel matrix: $256 \times 256$, voxel size: $1 \times 1 \times 1 \mathrm{~mm}$ ).

The cortical parcellation was performed using FreeSurfer (version 5.3) ${ }^{44}$. The segmentations and parcellations were visually inspected and outliers were examined using the ENIGMA protocol (http://enigma.ini.usc.edu/ protocols/imaging-protocols). Mean (of left and right hemisphere) cortical surface area and cortical thickness of the anterior and posterior insula (based on the Destrieux atlas ${ }^{45}$ ), rostral and caudal ACC, medial OFC (based on the Desikan-Killiany atlas ${ }^{46}$ ) and mean hippocampus, amygdala and ventral striatal volume (nucleus accumbens, based on the Fischl atlas ${ }^{47}$ ) were included as regions of interest (Supplementary Fig. S1).

\section{Data analysis}

\section{Symptom subtypes}

A $k$-means clustering in $\mathrm{R}$ was applied to the 16 depression items of the QIDS to identify symptom subtypes in the 275 young people diagnosed with MDD from the YoDA sample ${ }^{48}$. With $k$-means clustering, clusters are formed based on the cluster mean (centroid) that is 
Table 1 Demographics and clinical characteristics of symptom subtypes identified in the YoDA discovery sample.

\begin{tabular}{|c|c|c|c|c|c|}
\hline & SIA $(N=59)$ & SDA $(N=105)$ & MOD $(N=111)$ & $p$-value & Post hoc \\
\hline Age & $19.9(2.4)$ & $19.6(2.6)$ & $20.0(2.9)$ & 0.56 & \\
\hline Sex $(F), N(\%)$ & $43(73 \%)$ & 59 (56\%) & $59(53 \%)$ & 0.04 & $\mathrm{SIA}>\mathrm{SDA}, \mathrm{MOD}$ \\
\hline Comorbid ANX, N (\%) & $30(51 \%)$ & $62(59 \%)$ & $56(50 \%)$ & 0.75 & \\
\hline Age of onset MDD & $15.5(3.2)$ & $12.8(2.4)$ & $13.6(2.6)$ & 0.20 & \\
\hline Recurrent \% & 37.3 & 32.4 & 29.7 & 0.77 & \\
\hline Current AD use, $N(\%)$ & $2(3.4)$ & $11(10.4)$ & $5(4.5)$ & 0.12 & \\
\hline Lifetime AD use, $N(\%)$ & $7(11.9)$ & $17(16.2)$ & $15(13.5)$ & 0.41 & \\
\hline SSRI (N) & 5 & 14 & 12 & & \\
\hline SNRI (N) & 2 & 1 & 1 & & \\
\hline TCA (N) & 0 & 1 & 2 & & \\
\hline FH MDD \% & 52.5 & 43.8 & 45.0 & 0.36 & \\
\hline QIDS & $18.4(2.7)$ & $19.5(2.4)$ & $14.1(2.8)$ & $<0.001$ & $\mathrm{SDA}>\mathrm{SIA}>\mathrm{MOD}$ \\
\hline MADRS & $31.0(5.0)$ & $35.4(5.7)$ & $30.9(4.8)$ & $<0.001$ & $S D A>S I A, M O D$ \\
\hline GAD-7 & $14.7(4.6)$ & $15.4(4.9)$ & $10.0(4.9)$ & $<0.001$ & $\mathrm{MOD}<\mathrm{SIA}, \mathrm{SDA}$ \\
\hline SOFAS & $56.7(11.1)$ & $57.0(11.3)$ & $58.4(11.0)$ & 0.55 & \\
\hline BMI & $29.5(7.7)$ & $24.2(6.0)$ & $26.2(8.0)$ & $<0.001$ & $S I A>S D A, M O D$ \\
\hline Anorexia nervosa, $N(\%)$ & $1(2 \%)$ & $2(2 \%)$ & $3(3 \%)$ & 0.89 & \\
\hline Bulimia nervosa, N (\%) & $2(3 \%)$ & $3(3 \%)$ & $2(2 \%)$ & 0.80 & \\
\hline Binge eating disorder, $N(\%)$ & $9(15 \%)$ & $3(3 \%)$ & $1(1 \%)$ & $<0.001$ & $S I A>S D A, M O D$ \\
\hline
\end{tabular}

$A D$ antidepressant, $A N X$ anxiety disorder, $B M I$ body mass index, $F$ females, $F H$ family history, GAD-7 generalized anxiety disorder $7, M A D R S$ Montgomery Äsberg depression rating scale, $M D D$ major depressive disorder, $M O D$ moderate depression subtype, $N$ number of, QIDS quick inventory of depressive symptomatology, SDA severe depression with decreased appetite and insomnia subtype, SIA severe depression with increased appetite subtype, SOFAS social and occupational functioning assessment scale, SNRI serotonin and norepinephrine reuptake inhibitor, SSRI selective serotonin reuptake inhibitor, TCA tricyclic antidepressant.

Table 2 Demographics and clinical characteristics of symptom subtypes identified in the STAR*D replication sample.

\begin{tabular}{|c|c|c|c|c|c|}
\hline & SIA $(N=54)$ & SDA $(N=82)$ & MOD $(N=108)$ & $p$-value & Post hoc \\
\hline Age & $22.3(2.0)$ & $21.8(2.0)$ & $21.8(1.9)$ & 0.29 & \\
\hline Sex $(F), N(\%)$ & $42(78 \%)$ & $60(73 \%)$ & 75 (69\%) & 0.53 & \\
\hline Comorbid ANX, N (\%) & $5(9 \%)$ & $11(13 \%)$ & $16(14 \%)$ & 0.61 & \\
\hline Eating disorder, $N(\%)$ & $1(2 \%)$ & $1(1 \%)$ & $0(0 \%)$ & 0.41 & \\
\hline QIDS & $18.3(3.2)$ & $18.4(2.5)$ & $12.4(2.3)$ & $<0.001$ & $\mathrm{SIA}, \mathrm{SDA}>\mathrm{MOD}$ \\
\hline Hamilton & $21.1(4.4)$ & $24.4(5.2)$ & $19.8(4.0)$ & $<0.001$ & $\mathrm{SDA}>\mathrm{SIA}, \mathrm{MOD}$ \\
\hline Q-LES-Q & $38.8(10.7)$ & $38.6(12.6)$ & $50.2(13.4)$ & $<0.001$ & $\mathrm{SIA}, \mathrm{SDA}<\mathrm{MOD}$ \\
\hline WSAS & $26.0(7.4)$ & $25.3(7.1)$ & $17.5(7.2)$ & $<0.001$ & $\mathrm{SIA}, \mathrm{SDA}>\mathrm{MOD}$ \\
\hline
\end{tabular}

$A N X$ anxiety disorder, $F$ females, MOD moderate depression subtype, $N$ number of, QIDS quick inventory of depressive symptomatology, $Q-L E S-Q$ quality of life enjoyment and satisfaction questionnaire, SDA severe depression with decreased appetite and insomnia subtype, SIA severe depression with increased appetite subtype, WSAS work and social adjustment scale.

closest to a data point (the item score of a subject) to keep the centroids as small as possible. The QIDS item data (scored between 0 and 3) were scaled by subtracting the mean and dividing by the standard deviation to make sure all variables had the same weight.

\section{Selecting the optimal number of clusters}

The number of clusters $(k)$ was selected based on the highest number of partitioning methods in $\mathrm{R}$ that selected the same optimal number of clusters. The $\mathrm{R}$ package NbClust was used to determine the optimal number of 
clusters by looking at different combinations of distance measures and clustering methods based on hierarchical clustering (ward.D2, which minimizes variance within clusters) (for more details, please see Supplementary Material) $)^{49}$.

\section{K-means clustering}

The optimal number of clusters determined by NbClust was used as an input parameter to $K$-means clustering using the stats package in $\mathrm{R}^{48}$, to identify the centers of the clusters (centroids). To prevent the clustering settling on local minima, an initialization method was used to pick cluster means that covered the full range ${ }^{50,51}$. In this initialization method, random centers were selected, after which the procedure was reran to readjust the centers. The centroids of the next cluster were selected by maximizing the distance to the centroids that were selected before. These centers were used to run the $K$-mean clustering.

\section{Testing validity and stability}

Three methods were used to test the validity and reliability of the clusters. First, to test the stability of the clusters we repeated our clustering analysis in 10,000 randomly selected subsamples, each containing 100 participants from a pre-selected training sample (which consisted of $70 \%$ of the total sample). In each of the 10,000 subsamples, participants left out of the cluster identification process (the remaining $30 \%$ ) were assigned to clusters using linear discriminant analysis classifiers. The left-out sample was combined with the training sample to form a complete cluster solution. We then tested whether the individual cluster assignments were stable over the 10,000 subsamples by calculating an adjusted Rand score to test the similarity between each subsampling clustering solution compared to the original clustering solution. A Rand index of 1 means that the clustering solutions completely agree on the labels, while a Rand index of 0 represents a disagreement in the clustering. We also calculated the cluster-to-cluster index, which represents the mean distance between the clusters in the original and the new clustering obtained through resampling. Second, the optimal number of clusters was tested against a null distribution with permutation testing ${ }^{52}$. The same analysis procedure, including subsampling and permutation testing was repeated in the independent replication dataset STAR*D. A latent class analysis was performed to test the robustness of the $k$-means clustering method and to compare our findings to findings in previous adult studies that mainly employed latent class analysis (Supplementary Materials).

\section{Differences in clinical and structural brain characteristics between subtypes}

The symptom subtypes that were identified were compared on clinical and demographic characteristics in $\mathrm{R}$ using ANOVA or $\chi^{2}$ tests, and if there was a significant difference at $p<0.05$, post hoc tests with Tukey HSD correction for multiple comparisons (3 tests to compare the 3 subtypes).

In the YoDA sample, mean cortical surface area and thickness and subcortical volume of the regions of interest were compared between subtypes using an ANCOVA with group (MDD subtypes and healthy controls) as predictor and age and sex, as well as intercranial volume (ICV) as covariates. We did not include ICV as a covariate in analysis with cortical thickness measures, since thickness does not scale with head size ${ }^{53}$. False discovery rate (FDR) correction for 13 regions of interest was applied and Tukey HSD corrected post hoc tests were performed when a significant main effect of group was found. Analyses were repeated in an antidepressant naive sample to control for the possible effect of antidepressant use.

\section{Results}

\section{Symptom subtypes}

A 3-cluster solution was found to be the optimal fit according to 9 out of 26 partitioning methods (Supplementary Fig. S2). The stability of the clusters was tested for 2 as well as 3 clusters. The average Rand Index was 0.40 for 2 clusters, and 0.56 for 3 clusters. In addition, the average cluster-to-cluster distance was 1.35 for 2 clusters and 1.44 for 3 clusters, meaning that more labels agreed when 3 clusters were used and the means of different clusters were further apart in the 3-cluster solution. In addition, the Scott and Friedman partitioning measure showed that the index number for this number of clusters was higher than the cluster indices of an empirical null distribution, meaning that 3 clusters described the data better than data with no underlying clusters (Scott: $p<$ 0.001, Friedman: $p=0.01$, Supplementary Fig. S3).

The clusters were labeled as following: moderate depression $(n=111, \mathrm{MOD})$, severe depression with increased appetite $(n=59$, SIA) and severe depression with decreased appetite and insomnia $(n=105, \mathrm{SDA})$ (Fig. 1a). The MOD subtype endorsed symptoms such as a sad mood, lack of general interest, fatigue and typical neurovegetative symptoms of decreased appetite, weight loss and insomnia. The SIA and SDA subtypes both showed a higher severity of symptoms overall than the MOD subtype. The SIA subtype was uniquely characterized by endorsement of reversed (atypical) neurovegetative symptoms of increased appetite and weight gain, whereas the SDA subtype showed decreased appetite and higher levels of insomnia. The SIA subtype consisted of more females and was associated with higher BMI 

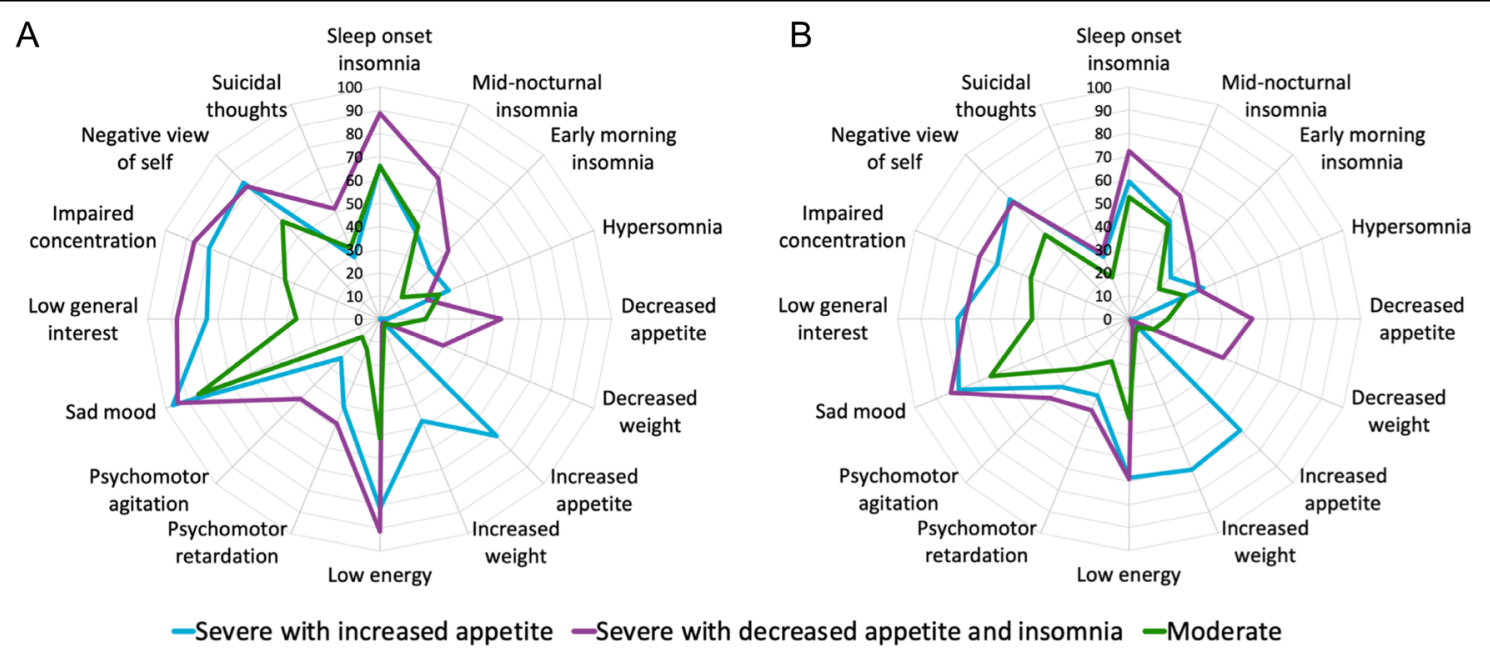

Fig. 1 Symptom subtypes in the YoDA discovery sample (a) and in a subsample of the STAR*D replication sample (b). A severe depression with increased appetite (SIA) subtype, severe depression with decreased appetite and insomnia (SDA) subtype and a moderate depression (MOD) subtype were identified in both datasets. The axis shows the percentage of subjects within a subtype that shows the symptoms in the radar plot (QIDS items).

compared to SDA and MOD (Table 1). Similar clusters were identified in the STAR*D dataset, including a moderate depression subtype (MOD, $n=108$ ), a severe depression with increased appetite and weight gain subtype (SIA, $n=$ 54) and a severe depression with decreased appetite and highest levels of insomnia subtype (SDA, $n=82$ ) (Fig. 1b, Table 2). The symptom clusters identified by the latent class analysis are displayed in Supplementary Fig. 4.

\section{Structural brain alterations in symptom subtypes}

The sample sizes of the subtypes with imaging data were 32, 49, 56 and 100 for SIA, SDA, MOD and HC, respectively. Anterior insula surface area showed a main effect of group ( $p_{\text {fdr-corrected }}<0.001$ ), which was driven by lower surface area in these regions in the SIA subtype compared to healthy controls (post hoc: $p=0.02$ ) (Fig. 2). Weak evidence was found for group differences in the rostral ACC surface area, anterior insula thickness, hippocampus and amygdala volume $\left(p_{\mathrm{fdr} \text {-corrected }}=0.08, p_{\mathrm{fdr} \text {-corrected }}=0.09\right.$, $p_{\mathrm{fdr}-\text { corrected }}=0.09$ and $p_{\mathrm{fdr} \text {-corrected }}=0.07$ respectively, Table 3). Although not significant, all three depressive subtypes showed lower surface area in the rostral ACC surface area and lower hippocampal volume compared to healthy controls. The SDA subtype had relatively lower anterior insula thickness, and the SIA subtype showed higher amygdala volume than $\mathrm{HC}$, whereas SDA showed lower amygdala volume compared to healthy controls. No group differences were found for the other regions of interest (Supplementary Table S1). Among the participants with neuroimaging data, the subtypes did not show differences in BMI (Supplementary Table S2). The results were replicated in a subset of the sample excluding lifetime antidepressant users. In the subset consisting of only

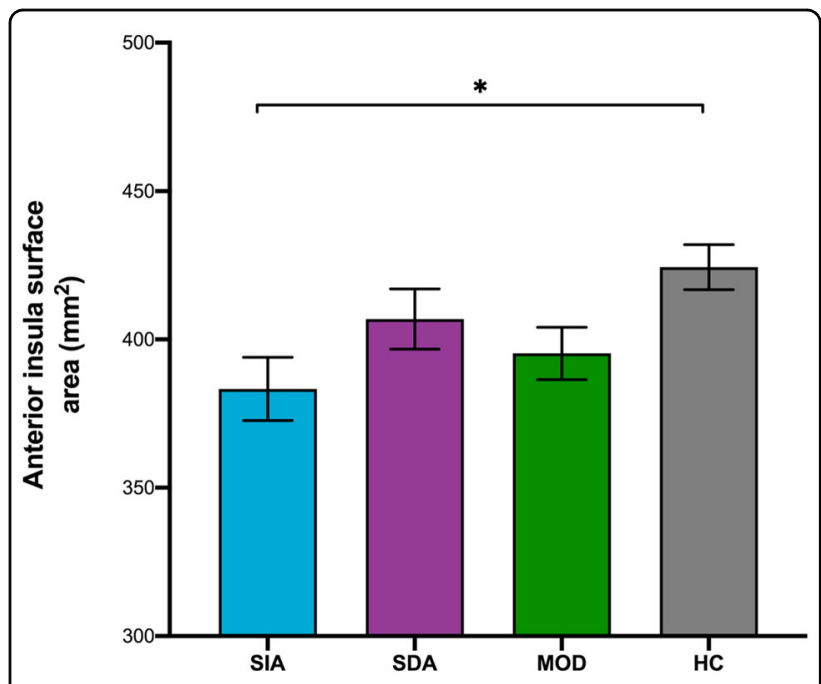

Fig. 2 Mean anterior insula surface area in the subtypes in the YoDA discovery sample. Severe increased appetite (SIA) subtype, severe decreased appetite and insomnia (SDA) subtype, moderate (MOD) subtype and healthy control (HC). SIA showed significantly lower surface area in the anterior insula compared to HC.

antidepressant naive patients $(n=119)$, the main effects for anterior insula surface area were still significant $\left(p_{\mathrm{fdr}-}\right.$ corrected $<0.001$ ), driven by differences between the SIA subtype and healthy controls $(p=0.01)$ (Supplementary Table S3 for the demographics).

\section{Discussion}

The aims of the current study were to identify datadriven subtypes of depressive symptoms in young people (aged 15-25) with MDD and to compare potential 
Table 3 Mean cortical surface area, thickness and subcortical volume in regions of interest per subtype.

\begin{tabular}{|c|c|c|c|c|c|c|}
\hline Region of interest & SIA & SDA & MOD & $\mathrm{HC}$ & $p_{\text {fdr-corrected }}$ & Post hoc \\
\hline \multicolumn{7}{|l|}{ Rostral ACC } \\
\hline Surface area & 741.80 & 736.84 & 746.51 & 783.49 & 0.08 & \\
\hline Thickness & 2.81 & 2.78 & 2.85 & 2.88 & 0.16 & \\
\hline \multicolumn{7}{|l|}{ Caudal ACC } \\
\hline Surface area & 711.63 & 698.62 & 703.62 & 729.23 & 0.25 & \\
\hline Thickness & 2.85 & 2.87 & 2.90 & 2.90 & 0.71 & \\
\hline \multicolumn{7}{|l|}{ Medial OFC } \\
\hline Surface area & 1856.38 & 1828.10 & 1894.59 & 1905.91 & 0.09 & \\
\hline Thickness & 2.43 & 2.45 & 2.47 & 2.46 & 0.51 & \\
\hline \multicolumn{7}{|l|}{ Anterior insula } \\
\hline Surface area & 383.31 & 406.91 & 395.33 & 424.42 & $0.001^{*}$ & $\mathrm{SIA}<\mathrm{HC}$ \\
\hline Thickness & 3.62 & 3.53 & 3.53 & 3.61 & 0.09 & \\
\hline \multicolumn{7}{|l|}{ Posterior insula } \\
\hline Surface area & 298.11 & 298.72 & 298.75 & 310.86 & 0.19 & \\
\hline Thickness & 3.48 & 3.46 & 3.44 & 3.51 & 0.51 & \\
\hline \multicolumn{7}{|l|}{ Hippocampus } \\
\hline Volume & 4433.28 & 4347.91 & 4431.89 & 4516.07 & 0.09 & \\
\hline \multicolumn{7}{|l|}{ Amygdala } \\
\hline Volume & 1801.67 & 1687.59 & 1773.73 & 1762.91 & 0.07 & \\
\hline \multicolumn{7}{|l|}{ Ventral striatum } \\
\hline Volume & 636.81 & 640.84 & 628.13 & 629.31 & 0.83 & \\
\hline
\end{tabular}

$H C$ healthy controls, MOD moderate depression subtype, SDA severe depression with decreased appetite and insomnia subtype, SIA severe depression with increased appetite subtype, *significant $p$-value.

structural brain alterations between these subtypes. The data-driven symptom subtypes found in the YoDA study cohort were in line with the subtypes characterized by opposite neurovegetative symptoms previously identified in adults ${ }^{11-18}$. One subtype showed atypical or reversed neurovegetative symptoms, mainly discriminated by increased appetite and weight gain, and two subtypes showed typical neurovegetative symptoms, including insomnia, decreased appetite and weight loss, with the typical symptom subtypes having different levels of overall severity (moderate versus severe). We replicated these data-driven symptom subtypes in a subsample of the STAR*D study, an independent sample of MDD patients within a similar age range. Symptom-based subtypes in young people have only been studied in one previous study in adolescents using latent class analysis, which identified similar subtypes ${ }^{18}$.

The data used in the current study are unique, as $34 \%$ of the MDD patients with imaging data were diagnosed with their first episode of MDD and 70\% were antidepressant naive. Identifying similar subtypes as found in adults in these more clinically specific (antidepressant free and at an early stage of the disorder) young people further validates the existence of the subtypes. Moreover, identifying similar subtypes in adolescents and young adults is relevant, as mood, appetite and sleep and their underlying biological processes go through developmental changes in adolescence, such as maturation of neural emotion regulation and reward processing circuitries, increasing levels of leptin, and a shift in the circadian rhythm ${ }^{54-56}$.

In line with previous adult studies, the increased appetite subtype had more females and a higher BMI than the other subtypes ${ }^{11,13,16,18}$. In addition, unlike some adult studies, the increased appetite subtype we identified was not discriminated by hypersomnia. However, hypersomnia items in a self-report questionnaire show low correlations with objective sleep measures ${ }^{57,58}$. In addition, whereas three items assess insomnia in the QIDS selfreport, only one item targets hypersomnia, and the disturbances might be more complex than assessed in that single question (for example fractionated or irregular night-time sleep but increased duration of sleep, including 
daytime napping). Therefore, sleep disturbances may still exist in the subtype with atypical neurovegetative symptoms. More ecologically valid assessments of sleep disturbances should be employed in future studies to examine sleep disturbances in these subtypes.

This study is the first to examine differences in structural brain alterations between data-driven symptombased subtypes. We found lower anterior insula surface area in the increased appetite subtype compared to healthy controls and the moderate severity subtype. Different parts of the insula are thought to have different roles, with the anterior insula important for integration of interoceptive information and reward and motivational processes $^{59}$. The anterior portion of the insula is preferentially interconnected with the orbitofrontal (OFC) and anterior cingulate cortices (ACC) and ventral striatum $^{60-62}$. Together with the dorsal ACC, the anterior insula forms a core hub of the so-called 'salience network', commonly implicated in interoceptive awareness; integrating external and internal stimuli to guide an individual's actions and decisions ${ }^{63-66}$. The anterior insula integrates information about the motility of the digestive system and hunger. Hormones (including leptin and insulin), body weight status and inflammation have been shown to influence insula activity and volume ${ }^{67-70}$. As alterations in hormones, such as leptin and insulin, and inflammatory markers have been observed in the increased appetite subtype in adults and those hormones and inflammatory markers have been found to affect surface area $^{71}$, is possible that alterations in these endocrine factors that may be unique to this atypical neurovegetative subtype are related to surface area alterations.

Furthermore, the insula is implicated in reward processing and emotion regulation, processes that have been associated with food intake ${ }^{72-75}$. In addition, insula alterations are found to be associated with eating disorders $^{76}$. Previous research reported increased brain activity in the anterior insula and other reward regions including the ventral striatum in response to pictures of food in adult MDD patients with increased appetite ${ }^{26}$. In addition, emotion regulation disturbances have shown to increase emotional eating ${ }^{77}$, which may underlie the increased appetite and weight gain observed in the atypical neurovegetative subtype, potentially mediated by structural alterations in the anterior insula ${ }^{78}$.

Only two prior studies in adults examined differences in brain measures between MDD patients selected on the presence of depression-related symptoms of increased appetite versus decreased appetite ${ }^{21,26}$. These studies examined neural responses during an fMRI food picture task, and found that lower ventral striatum activity was associated with higher cortisol in the decreased appetite subtype. In contrast, in the increased appetite subtype higher anterior insula activity was observed. In line with these studies by Simmons et al., we found anterior insula surface area alterations in the increased appetite subtype. However, no differences in ventral striatum volume between the subtypes were found in the present study, suggesting that alterations in the ventral striatum might be restricted to a functional level.

Interestingly, only surface area differences were observed for the anterior insula and no differences were found in insular cortical thickness. Cortical surface area and cortical thickness are two distinct characteristics of the brain's cortex and have different developmental pathways. Cortical thickness increases until approximately age 2, whereas cortical surface area increases, depending on the region, until adolescence, making it more vulnerable to early life stressors ${ }^{79-82}$. In addition, cortical surface area alterations have been found to be associated with early onset depression ${ }^{83,84}$, and prior research shows that the increased appetite, or atypical neurovegetative, subtype is associated with earlier onset of depression $^{13,19,24}$. However, as this study consisted of adolescents and young adults, the age of onset was low overall and did not differ between subtypes.

A few limitations of the study should be noted. The exclusion criteria of the YoDA studies might have influenced clustering results, and compromise generalizability. Only young people with MDD who showed moderate to severe depressive symptoms were included, therefore not representing the whole depressive spectrum. In addition, the $k$-means clustering might have been affected by the high negative correlations between the increased appetite/ weight and decreased appetite/weight symptoms, and between insomnia and hypersomnia. There has been some critique regarding the subtyping based on symptoms including these opposite neurovegetative symptoms using a latent class analysis or other data-driven techniques, since they are complete opposites and one symptom automatically rules out the possibility of endorsing the other symptom (e.g., a person can not endorse both weight gain and weight loss at the same time, although they can endorse no changes in weight). The negative correlations between increased and decreased appetite $(-0.49)$ and weight $(-0.31)$, and between insomnia and hypersomnia $(-0.14$ to -0.21$)$, known as a violation of conditional independence, have likely dominated the clustering and may have masked subtypes based on other patterns of symptom endorsement ${ }^{85}$. However, differences in genetics, blood markers of inflammation, leptin insensitivity and insulin resistance, and neuroimaging markers have been repeatedly found between these subtypes derived using a data-driven analysis ${ }^{19,20,24,25,78}$ and the current study, and when selected on the presence of increased versus decreased appetite ${ }^{21,26,86}$. Therefore, the subtypes seem biologically relevant. Another limitation of using $k$-means clustering is that $k$ (number of clusters) has 
to be chosen manually, however, this process is made as transparent as possible by showing all the steps taken to decide the optimal value for $k$. On the other hand, advantages of $k$-means clustering are that it identifies clusters that do not have to have the same size and shape. Finally, unfortunately STAR*D did not have imaging data available, and we were therefore not able to validate the imaging findings in an independent sample.

This clinical relevance of these neurovegetative symptom subtypes is underlined by the persistence of sleep and appetite disturbances after treatment for depression ${ }^{87,88}$. In addition, these neurovegetative symptoms do not affect clinical management decisions as much as mood symptoms, even though they are associated with high risks of suicide $^{89}$, obesity and metabolic syndrome ${ }^{90}$, and depression recurrence ${ }^{91-93}$. Subtyping depression based on neurovegetative symptoms could lead to more targeted intervention. Even though we had treatment outcome data for a subset of the YoDA sample and the STAR*D subsample, the sample sizes for each subtype per treatment type were too small to evaluate differences in response to treatment between subtypes in these samples. Future studies with larger sample sizes that are properly powered for detecting differences in treatment response between subtypes of depression are needed to elucidate whether young people with an atypical neurovegetative subtype depression show a different response to traditional treatments (such as antidepressants or psychotherapy) or novel treatments compared to those with a more typical profile of neurovegetative symptoms. Our findings of structural alterations in the anterior insula together with previous findings of functional alterations in the insula uniquely associated with the atypical neurovegetative subtype suggest that core functions of the insula including interoceptive function, emotion regulation and reward processing may be promising treatment targets for this specific subtype of depression.

To conclude, we were able to replicate the existence of reversed neurovegetative and typical neurovegetative symptom subtypes of depression in two adolescent/young adult MDD samples. This was the first study to show that these symptom subtypes were associated with cortical surface alterations in the anterior insula, with the increased appetite showing lower surface area compared to the moderate subtype and healthy controls. Together with previous findings in adults, our current findings suggest that the subtype with atypical neurovegetative symptoms may have a unique biological signature. Moreover, neurovegetative symptoms are associated with poorer clinical outcomes and antidepressant treatment has shown to work more effectively for mood and cognitive symptoms than for atypical and sleep symptoms $^{94,95}$. Therefore, these neurovegetative symptoms subtypes, characterized by changes in sleep and appetite, should be noted when treating young people with depression.

\section{Acknowledgements}

This study was supported by the National Health and Medical Research Council of Australia (NHMRC) Project Grants to C.G.D. (YoDA C ID 1024570), M. B. (YoDA A ID 1027315) and B.J.H. (1064643). B.J.H. is supported by a NHMRC Career Development Fellowship (1124472). C.G.D. is supported by an NHMRC Career Development Award (141738). M.B. is supported by a National Health and Medical Research Council (NHMRC) Senior Principal Research Fellowship (APP1059660 and APP1156072). L.S. is supported by a NHMRC Career

Development Fellowship (1140764) and gratefully acknowledges support from The Netherlands Brain Foundation (F2014[1]-24) for this work.

\section{Author details}

'Orygen, The National Centre of Excellence in Youth Mental Health, Parkville, VIC, Australia. ${ }^{2}$ Centre for Youth Mental Health, The University of Melbourne, Parkville, VIC, Australia. ${ }^{3}$ Melbourne Neuropsychiatry Centre, Department of Psychiatry, The University of Melbourne \& Melbourne Health, Melbourne, VIC, Australia. ${ }^{4}$ Department of Psychiatry, Amsterdam UMC, Amsterdam, The Netherlands. ${ }^{5}$ Donders Institute for Brain, Cognition and Behaviour, Radboud University, Nijmegen, The Netherlands. 'IMPACT SRC, School of Medicine, Barwon Health, Deakin University, Geelong, VIC, Australia. ${ }^{7}$ Department of Psychiatry, The University of Melbourne, Melbourne, VIC, Australia. ${ }^{8}$ Florey Institute of Neuroscience and Mental Health, The University of Melbourne, Parkville, VIC, Australia

Conflict of interest

The authors declare that they have no conflict of interest.

\section{Publisher's note}

Springer Nature remains neutral with regard to jurisdictional claims in published maps and institutional affiliations.

Supplementary Information accompanies this paper at (https://doi.org/ 10.1038/s41398-020-0787-9).

Received: 5 November 2019 Revised: 17 February 2020 Accepted: 26 February 2020

Published online: 20 April 2020

\section{References}

1. American Psychiatric Association. Diagnostic and Statistical Manual of Mental Disorders 5th ed. (Author, Washington, DC, 2013).

2. Gore, F. M. et al. Global burden of disease in young people aged 10-24 years: a systematic analysis. Lancet 377, 2093-2102 (2011).

3. Zisook, S. et al. Effect of age at onset on the course of major depressive disorder. Am. J. Psychiatry 164, 1539-1546 (2007).

4. Kessler, R. C. et al. Lifetime prevalence and age-of-onset distributions of DSMIV disorders in the National Comorbidity Survey Replication. Arch. Gen. Psychiatry 62, 593-602 (2005).

5. March, J. et al. Fluoxetine, cognitive-behavioral therapy, and their combination for adolescents with depression. JAMA 292, 807-820 (2004).

6. Kennard, B. D. et al. Remission and recovery in the treatment for adolescents with depression study (TADS): acute and long-term outcomes. J. Am. Acad. Child Adolesc. Psychiatry 48, 186-195 (2009).

7. Østergaard, S. D., Jensen, S. O. W. \& Bech, P. The heterogeneity of the depressive syndrome: When numbers get serious. Acta Psychiatr. Scand. 124, 495-496 (2011).

8. Parker, G. et al. Atypical depression: a reappraisal. Am. J. Psychiatry 159, 1470-1479 (2002).

9. Posternak, M. A. \& Zimmerman, M. Partial validation of the atypical features subtype of major depressive disorder. Arch. Gen. Psychiatry 59, 70-76 (2002).

10. Li, Y. et al. Subtypes of major depression: latent class analysis in depressed Han Chinese women. Psychol. Med. 44, 3275-3288 (2015). 
11. Lamers, F. et al. Identifying depressive subtypes in a large cohort study: results from the Netherlands Study of Depression and Anxiety (NESDA). J. Clin. Psychiatry 71, 1582-1589 (2010).

12. Ulbricht, C. M., Rothschild, A. J. \& Lapane, K. L. The association between latent depression subtypes and remission after treatment with citalopram: a latent class analysis with distal outcome. J. Affect Disord. 188, 270-277 (2015).

13. Veltman, E. M. et al. Depressive subtypes in an elderly cohort identified using latent class analysis. J. Affect Disord. 218, 123-130 (2017).

14. Rodgers, S. et al. Symptom-based subtypes of depression and their psychosocial correlates: a person-centered approach focusing on the influence of sex. J. Affect Disord. 156, 92-103 (2014).

15. Sullivan, P. F., Prescott, C. A. \& Kendler, K. S. The subtypes of major depression in a twin registry. J. Affect Disord. 68, 273-284 (2002)

16. Alexandrino-Silva, C. et al. Gender differences in symptomatic profiles of depression: Results form the Sao Paulo Megacity Mental Health Survey. J. Affect Disord. 147, 355-364 (2013).

17. Sullivan, P. F., Kessler, R. C. \& Kendler, K. S. Latent class analysis of lifetime depressive symptoms in the National Comorbidity Survey. Am. J. Psychiatry 155, 1398-1406 (1998).

18. Lamers, F. et al. Structure of major depressive disorder in adolescents and adults in the US general population. Br. J. Psychiatry 201, 143-150 (2012).

19. Lamers, F. et al. Evidence for a differential role of HPA-axis function, inflammation and metabolic syndrome in melancholic versus atypical depression. Mol. Psychiatry 18, 692-699 (2013).

20. Milaneschi, Y., Lamers, F., Bot, M., Drent, M. L. \& Penninx, B. W. J. H. Leptin dysregulation is specifically associated with major depression with atypical features: evidence for a mechanism connecting obesity and depression. Biol. Psychiatry 81, 807-814 (2017).

21. Simmons, W. K. et al. Appetite changes reveal depression subgroups with distinct endocrine, metabolic, and immune states. Mol. Psychiatry. https://doi. org/10.1038/s41380-018-0093-6 (2018).

22. Caroleo, M., et al. The role of hormonal, metabolic and inflammatory biomarkers on sleep and appetite in drug free patients with major depression: a systematic review. J. Affect Disord. 250, 249-259 (2019).

23. Brailean, A., Curtis, J., Davis, K., Dregan, A., \& Hotopf, M. Characteristics, comorbidities, and correlates of atypical depression: evidence from the UK Biobank Mental Health Survey. Psychol. Med. 1-10 (2019).

24. Milaneschi, Y. et al. Genetic association of major depression with a typical features and obesity-related immunometabolic dysregulations. JAMA Psychiatry 74, 1214-1225 (2017).

25. Milaneschi, Y. et al. Polygenic dissection of major depression clinical heterogeneity. Mol. Psychiatry 21, 516-522 (2016).

26. Simmons, K. et al. Depression-related increases and decreases in appetite reveal disssociable patterns of abberant activity in reward and interoceptive neurocircuitry. Am. J. Psychiatry 173, 418-428 (2016).

27. Cosgrove, K. T. et al. Appetite change profiles in depression exhibit differential relationships between systemic inflammation and activity in reward and interoceptive neurocircuitry. Brain Behav. Immun. 83, 163-171 (2020).

28. Berridge, K. C. 'Liking' and 'wanting' food rewards: brain substrates and roles in eating disorders. Physiol. Behav. 97, 537-550 (2010).

29. Martin, L. E. et al. Neural mechanisms associated with food motivation in obese and healthy weight adults. Obesity 18, 254-260 (2010).

30. Rolls E. T. Functions of the orbitofrontal and pregenual cingulate cortex in taste, olfaction, appetite and emotion. Acta Physiol. Hungarica 95, 131-164 (2008).

31. Tracy, A. L., Jarrard, L. E. \& Davidson, T. L. The hippocampus and motivation revisited: appetite and activity. Behav. Brain Res. 127, 13-23 (2001)

32. Davey, C. G. et al. The addition of fluoxetine to cognitive behavioural therapy for youth depression (YoDA-C): a randomised, double-blind, placebo-controlled, multicentre clinical trial. Lancet Psychiatry. 6, 735-744 (2019).

33. Quin, A. L. et al. Youth depression alleviation-augmentation with an antiinflammatory agent (YoDA-A): Protocol and rationale for a placebo-controlled randomised trial of rosuvastatin and aspirin. Early Interv. Psychiatry 12, 45-54 (2018).

34. First, M. B., Spitzer, R. L., Gibbon, M. \& Williams, J. B. W. Structured Clinical Interview for DSM-IV Axis I Disorders (SCID-I) (American Psychiatric Publishing, Washington, DC, US, 1997).

35. Hamilton, M. A rating scale for depression. J. Neurol. Neurosurg. Psychiatry $\mathbf{2 3}$ 56-62 (1960)

36. Rush, A. J. et al. The 16-item quick inventory of depressive symptomatology. Depression 54, 573-583 (2003).
37. Montgomery, S. A. \& Asberg, M. A new depression scale designed to be sensitive to change. Br. J. Psychiatry 134, 382-389 (1979).

38. Babor, T., Higgins-Biddle, J. C., Saunders, J. B., \& Monteiro, M. G. The Alcohol Use Disorders Identification Test: Guidelines for Use in Primany Care 1-40 (WHO, Geneva, 2001).

39. Morosini, P. L., Magliano, L., Brambilla, L., Ugolini, S. \& Pioli, R. Development, reliability and acceptability of a new version of the DSM-IVSocial and Occupational Functioning Assessment Scale (SOFAS) to assessroutine social functioning. Acta Psychiatr. Scand. 101, 323-329 (2000).

40. Spitzer, R. L., Kroenke, K., Williams, J. B. W. \& Lowe, B. A brief measure for assessing generalized anxiety disorder. Arch. Intern. Med. 166, 1092-1097 (2006).

41. Zimmerman, M. \& Mattia, J. I. A self-report scale to help make psychiatric diagnoses. Arch. Gen. Psychiatry 58, 787-794 (2001).

42. Endicott, J., Nee, J., Harrison, W., \& Blumenthal, R. Quality of life enjoyment and satisfaction questionnaire: a new measure. Psychopharmacol. Bull. 29, 321-326 (1993).

43. Mundt, J. C., Marks, I. M., Shear, M. K. \& Greist, J. M. The work and social adjustment scale a simple measure of impairment in functioning. Br. J. Psychiatry 180, 461-464 (2002).

44. Dale, A. M., Fischl, B. \& Sereno, M. I. Cortical surface-based analysis. I. Segmentation and surface reconstruction. Neuroimage 9, 179-194 (1999).

45. Destrieux, C., Fischl, B., Dale, A. \& Halgren, E. A sulcul depth-based anatomical parcellation of the cerebral cortex. Neuroimage 47, S151 (2009).

46. Desikan, R. S. et al. An automated labeling system for subdividing the human cerebral cortex on MRI scans into gyral based regions of interest. Neuroimage 31, 968-980 (2006)

47. Fischl, B. et al. Whole brain segmentation. Neuron 33, 341-355 (2002).

48. R Core Team. R: A Language and Environment for Statistical Computing (R Foundation for Statistical Computing: Vienna, Austria, 2019). https://www.r project.org/.

49. Charrad, M., Ghazzali, N., Boiteau, V., Niknafs, A. NbClust: an R package for determining the relevant number of clusters in a data set. J. Stat. Softw. $\mathbf{6 1}$ 1-36. http://www.jstatsoft.org/v61/i06/ (2014).

50. Rogerson, D. Assessing stability of K-means clusterings. http:// activisiongamescience.github.io/2016/08/19/Assessing-Stability-of-K-MeansClusterings/ (2016).

51. Luxburg, U. Von. Clustering stability: an overview. Found. Trends Mach. Learn. 2 235-274 (2010).

52. Dinga, R. et al. Evaluating the evidence for biotypes of depression: methodological replication and extension of Drysdale et al. (2017). Neurolmage Clin. 22, 101796 (2019).

53. Barnes, J. et al. Head size, age and gender adjustment in MRI studies: a necessary nuisance? Neuroimage 53, 1244-1255 (2010).

54. Hagenauer, M. H., Perryman, J. I., Lee, T. M. \& Carskadon, M. A. Adolescent changes in the homeostatic and circadian regulation of sleep. Dev. Neurosci. 31, 276-284 (2009)

55. Maxwell, M. A. \& Cole, D. A. Weight change and appetite disturbance as symptoms of adolescent depression: toward an integrative biopsychosocial model. Clin. Psychol. Rev. 29, 260-273 (2009).

56. Ong, K. K. L., Ahmed, M. L. \& Dunger, D. B. The role of leptin in human growth and puberty. Acta Paediatr. 88, 95-98 (1999).

57. Ohayon, M. M. \& Roberts, L. W. Challenging the validity of the association between oversleeping and overeating in atypical depression. J. Psychosom. Res. 78, 52-57 (2015).

58. Meliska, C. J. et al. Relationship of morningness-eveningness questionnaire score to melatonin and sleep timing, body mass index and atypical depressive symptoms in peri- and post-menopausal women. Psychiatry Res. 188, 88-95 (2011).

59. Simmons, W. K. et al. Keeping the body in mind: Insula functional organization and functional connectivity integrate interoceptive, exteroceptive, and emotional awareness. Hum. Brain Mapp. 34, 2944-2958 (2013).

60. Augustine, J. R. Circuitry and fimctional aspects of the insular lobe in primates including humans. Brain Res. Rev. 22, 229-244 (1996).

61. Chikama, M., McFarland, N. R., Amaral, D. G. \& Haber, S. N. Insular cortical projections to functional regions of the striatum correlate with cortical cytoarchitectonic organization in the primate. J. Neurosci. 17, 9686-9705 (1997).

62. Mesulam, M. \& Mufson, E. J. Insula of the Old World Monkey I: architectonics in the Insulo-orbito- temporal Component of the paralimbic brain. J. Comp. Neurol. 22, 1-22 (1982). 
63. Menon, V. \& Uddin, L. Q. Saliency, switching, attention and control: a network model of insula function. Brain Struct. Funct. 214, 655-667 (2010).

64. Sridharan, D., Levitin, D. \& Menon, V. A critical role for the right fronto-insular cortex in switching between central-executive and default-mode networks. Proc. Natl Acad. Sci. USA 105, 12569-12574 (2008).

65. Craig, A. D. How do you feel-now? The anterior insula and human awareness. Nat. Rev. Neurosci. 10, 59-70 (2009).

66. Craig, A. D. Interoception: the sense of the physiological condition of the body. Curr. Opin. Neurobiol. 13, 500-505 (2003).

67. De Silva, A., Salem, V., Matthews, P. M., \& Dhillo, W. S. The use of functional MRI to study appetite control in the CNS. Exp. Diabetes Res. 2012, 764017 (2012).

68. Malik, S., McGlone, F., Bedrossian, D. \& Dagher, A. Ghrelin modulates brain activity in areas that control appetitive behavior. Cell Metab. 7, 400-409 (2008).

69. Schloeg, H., Percik, R., Horstmann, A., Villringer, A. \& Stumvoll, M. Peptide hormones regulating appetite-focus on neuroimaging studies in humans. Diabetes Metab. Res. Rev. 27, 104-112 (2011).

70. Smucny, J. et al. Brain structure predicts risk for obesity. Appetite 59, 859-865 (2012).

71. Marsland, A. L. et al. Brain morphology links systemic inflammation to cognitive function in midlife adults. Brain Behav. Immun. 48, 195-204 (2015).

72. Naqvi, N. \& Bechara, A. The hidden island of addiction: the insula. Trends Neurosci. 32, 56-67 (2009).

73. Kohn, N. et al. Neural network of cognitive emotion regulation-An ALE meta- analysis and MACM analysis. Neuroimage 87, 345-355 (2014).

74. Evers, C., Stok, F. \& de Ridder, D. T. D. Feeding your feelings: emotion regulation strategies and emotional eating. Personal. Soc. Psychol. Bull. 36 792-804 (2010).

75. Fulton, S. Appetite and reward. Front. Neuroendocrinol. 31, 85-103 (2010).

76. Frank, G. K. W. Altered brain reward circuits in eating disorders: chicken or egg? Curr. Psychiatry Rep. 15, 1-11 (2013).

77. Wiser, S. \& Telch, C. F. Dialectical behavior therapy for binge-eating disorder. J. Clin. Psychol. 55, 7550-7768 (1999).

78. Shriver, L. H. et al. Longitudinal associations between emotion regulation and adiposity in late adolescence: indirect effects through eating behaviors. Nutrients 11, E517 (2019).

79. Amlien, I. K. et al. Organizing principles of human cortical developmentthickness and area from 4 to 30 years: insights from comparative primate neuroanatomy. Cereb. Cortex. 26, 257-267 (2016).

80. Ducharme, $\mathrm{S}$. et al. Trajectories of cortical thickness maturation in normal brain development - The importance of quality control procedures. Neuroimage 125, 267-279 (2016)
81. Tamnes, C. K. et al. Development of the cerebral cortex across adolescence: a multisample study of inter-related longitudinal changes in cortical volume, surface area, and thickness. J. Neurosci. 37, 3402-3412 (2017).

82. Wierenga, L. M., Langen, M., Oranje, B. \& Durston, S. Unique developmental trajectories of cortical thickness and surface area. Neuroimage 87, 120-126 (2014).

83. Schmaal, L. et al. Cortical abnormalities in adults and adolescents with major depression based on brain scans from 20 cohorts worldwide in the ENIGMA Major Depressive Disorder Working Group. Mol. Psychiatry 22, 900-909 (2017).

84. Schmaal, L. et al. Brain structural signatures of adolescent depressive symptom trajectories: A Longitudinal Magnetic Resonance Imaging Study. J. Am. Acad. Child Adolesc. Psychiatry 56, 593-601 (2017).

85. van Loo, H. M., Wanders, R. B. K., Wardenaar, K. J., \& Fried, E. I. Problems with latent class analysis to detect data-driven subtypes of depression. Mol. Psychiatry 23, 495-496 (2016).

86. Lamers, F., Milaneschi, Y., De Jonge, P., Giltay, E. J. \& Penninx, B. W. J. H. Metabolic and inflammatory markers: Associations with individual depressive symptoms. Psychol. Med. 48, 1102-1110 (2017).

87. Nierenberg, A. A. et al. Residual symptoms after remission of major depressive disorder with citalopram and risk of relapse: a STAR*D report. Psychol. Med. 40, 41-50 (2018).

88. Taylor, D. J., Walters, H. M., Vittengl, J. R., Krebaum, S. \& Jarrett, B. Cognitive therapy of depression and predict relapse and and recurrance. J. Affect Disord. 123, 181-187 (2011).

89. McGirr, A. et al. An examination of DSM-IV depressive symptoms and risk for suicide completion in major depressive disorder: a psychological autopsy study. J. Affect Disord. 97, 203-209 (2007).

90. Lamers, F., Beekman, A. T. F., van Hemert, A. M., Schoevers, R. A. \& Penninx, B. W. J. H. Six-year longitudinal course and outcomes of subtypes of depression. Br. J. Psychiatry 208, 62-68 (2016).

91. Piek, E. et al. Determinants of (non-)recognition of depression by general practitioners. J. Affect Disord. 138, 397-404 (2012).

92. Riepe, M. W., Gritzmann, P. \& Brieden, A. Preferences of psychiatric practitioners for core symptoms of major depressive disorder: a hidden conjoint analysis. Int J. Methods Psychiatr. Res. 26, 1-7 (2017).

93. Ohayon, M. M. \& Roth, T. Place of chronic insomnia in the course of depressive and anxiety disorders. J. Psychiatr. Res. 37, 9-15 (2003).

94. Thase, M. E. Atypical depression: useful concept, but it's time to revise the DSM-IV criteria. Neuropsychopharmacology 34, 2633-2641 (2009).

95. Chekroud, A. M. et al. Reevaluating the efficacy and predictability of antidepressant treatments. JAMA Psychiatry 74, 370-378 (2017). 